Int. J. Dev. Biol. 54: 1139-1149 (2010)

doi: $10.1387 / \mathrm{ijdb} .093055 \mathrm{ac}$

\title{
Genetic control of hematopoietic development in Xenopus and zebrafish
}

\author{
ALDO CIAU-UITZ\#,1, FENG LIU\#,2 and ROGER PATIENT*,1 \\ ${ }^{1}$ MRC Molecular Haematology Unit, Weatherall Institute of Molecular Medicine, University of Oxford, UK \\ and ${ }^{2}$ State Key Laboratory of Biomembrane and Membrane Biotechnology, Institute of Zoology, Chinese \\ Academy of Sciences, Beijing, P.R.China.
}

\begin{abstract}
Blood development has been highly conserved during evolution. Hematopoietic cells in amphibian and fish embryos, as in mammalian embryos, emerge and progressively differentiate in several locations. Hematopoiesis, including of the immune system, is similar in the amphibian, Xenopus, to mammals and the embryos are ideal for tissue transplantation and lineage labelling experiments, which have enabled the elucidation of the distinct origins of embryonic and adult hematopoietic cells, as well as their migration pathways and organ colonisation behaviours. The zebrafish hematopoietic system is less well understood, but these embryos have recently emerged as a powerful system for both genetic analysis and imaging. In this review, we summarise our current knowledge of the cellular and genetic basis of ontogeny of the hematopoietic system in Xenopus and zebrafish embryos.
\end{abstract}

KEY WORDS: hematopoiesis, hemangioblast, transcriptional regulation, adult hematopoietic stem cell

\section{Introduction}

Evolutionary conservation of hematopoietic development means that amenable model organisms, such as amphibia and fish, can be exploited to elucidate mechanism. Due to oxygen diffusion from the surrounding environment, fish and frog embryos are less hypoxic and therefore less dependent on a fully functional cardiovascular system, thus allowing the study of factors that cause embryonic arrest in more oxygen dependent embryos. Xenopus and zebrafish embryos are easy to culture and their large number and size allows extensive experimental manipulation. Genetic pathways can be readily studied using a variety of techniques to perturb them. Blood development in Xenopusis very similar to that in mammals, including a similarly developed immune system (reviewed by Du Pasquier et al., 2000). In addition, Xenopus embryos can be used to generate fate maps and for transplantation between cytogenetically distinct individuals. Over the last decade or so, the zebrafish system has emerged as a powerful genetic model in which to study blood development. Zebrafish are well suited to saturation mutagenesis and also imaging of cell fates and movements, often using transgenic reporter lines. Studies in these two model organisms are contributing significantly to our knowledge of developmental hematopoiesis.
In order to understand the genetics of hematopoietic development, it is essential to know the cellular hierarchies. We need to identify all the intermediate cell types and determine their locations as development takes place. This knowledge can provide clues as to the signals that the cells might be exposed to, and it also enables us to determine which particular combinations of transcription factors are competing for dominance in a given nucleus at any given time.

\section{Hematopoietic development in Xenopus}

\section{The ventral blood island (VBI)}

In Xenopus embryos, the first hematopoietic cells (erythrocytes) differentiate in the ventral blood island (VBI). The VBI is the ventralmost tissue in the Xenopus tadpole and therefore blood has been generally regarded as a ventral mesoderm derivative. In

Abbreviations used in this paper: ALM, anterior lateral plate mesoderm; CHT, caudal hematopoietic tissue; DA, dorsal aorta; DLP, dorsal lateral plate; DMZ, dorsal marginal zone; ETS, E-Twenty Six; GRN, genetic regulatory network; HSC, hematopoietic stem cell; PLM, posterior lateral plate mesoderm; TF, transcription factor; VBI, ventral blood islands; VMZ, ventral marginal zone.

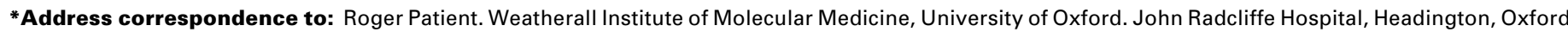
OX3 9DS, UK. e-mail: roger.patient@imm.ox.ac.uk Tel/Fax: +44-18-6522-2613/+44-18-6522-2501
}

\#These authors contributed equally to this article

Final, author-corrected PDF published online: 16 July 2010.

ISSN: Online 1696-3547, Print 0214-6282 
agreement, when incubated to the tail bud stage, only ventral marginal zone (VMZ, prospective ventral mesoderm) and not dorsal marginal zone (DMZ, prospective dorsal mesoderm) explants give rise to erythrocytes. This view has recently been challenged following the discovery of a dorsal mesoderm contribution to the VBI (Ciau-Uitz et al., 2000; Lane and Smith, 1999; Tracey etal., 1998; Walmsley et al., 2002). The first evidence was provided by explants cultured for less time, to neurula instead of tailbud stages. At neurula stages, the DMZ rather than the VMZ expressed blood genes (Tracey et al., 1998). Furthermore, injection of a lineage tracer into the marginal zone of 4-cell embryos conclusively demonstrated that the $\mathrm{DMZ}$ contributes to the most anterior portion of the $\mathrm{VBI}$, the aVBI, whereas the $\mathrm{VMZ}$ contributes to the posterior portion, the pVBI (Tracey etal., 1998). This finding was confirmed by fate mapping blastomeres of the 32-cell embryo which showed that the aVBI derives from the dorsal blastomeres, $\mathrm{C} 1$ and $\mathrm{D} 1$, and the $\mathrm{pVBI}$ derives from the ventral blastomere, D4, in regularly dividing embryos (Ciau-Uitz etal., 2000; Figs. 1A, 2A).

Gene expression in the precursors of the aVBI is first detected just after gastrulation in a population of hemangioblasts located in the most anterior ventral mesoderm (Tracey etal., 1998; Walmsley et al., 2002; Fig. 1B). Before gastrulation, these cells are located in the BMP deficient dorsal blastopore lip. However, as gastrulation proceeds they involute to become part of the mesodermal leading edge which migrates under the roof of the blastocoel (bcl). Finally, at the end of gastrulation they meet up with the ventral mesodermal leading edge, along the way encountering BMP which activates expression of hematopoietic and endothelial genes (Kumano et al., 1999; Walmsley et al., 2002; Fig. 1B). The aVBI precursors maintain their hemangioblast character until the end of neurulation (Fig. 1C), after which endothelial, myeloid and erythroid populations segregate as they differentiate. This anterior, embryonic hemangioblast population gives rise to a minority of the VBI erythrocytes but to a large number of myeloid cells (Costa et al., 2008; Tashiro et al., 2006; Walmsley et al., 2008; Fig. 1D).

The $\mathrm{pVBI}$ gives rise to early and late larval erythrocytes, leukocytes and a few, short term lymphoid cells (T cells) (Maeno et al., 1985b; Rollins-Smith and Blair, 1990; Smith and Turpen, 1985; Turpen et al., 1997). However, hematopoietic development in the pVBI differs from that in the aVBI. For example, while the aVBI derives from hemangioblast precursors, these have not yet been found for the pVBI (Walmsley et al., 2002; Walmsley et al., 2008). Furthermore, hematopoietic gene expression in the pVBI initiates long after that in the aVBI, a delay caused at least in part by FGF signalling (Walmsley et al., 2008). Thus, genes associated with erythroid differentiation, such as globin, are expressed first in the aVBI and only later in the pVBI. Similarly, myeloid differentiation in the $\mathrm{pVBI}$ or explanted VMZ occurs only after myelopoiesis in the aVBI or explanted DMZ has taken place, with the aVBI generated myeloid cells patrolling the tadpole, while myeloid cells from the pVBI are not seen until the early larval stages, after initiation of heart beating (Tashiro et al., 2006; Fig. 2 B-C).

The absence of a pVBI hemangioblast may suggest an interesting parallel with the mouse yolk sac, which gives rise to two

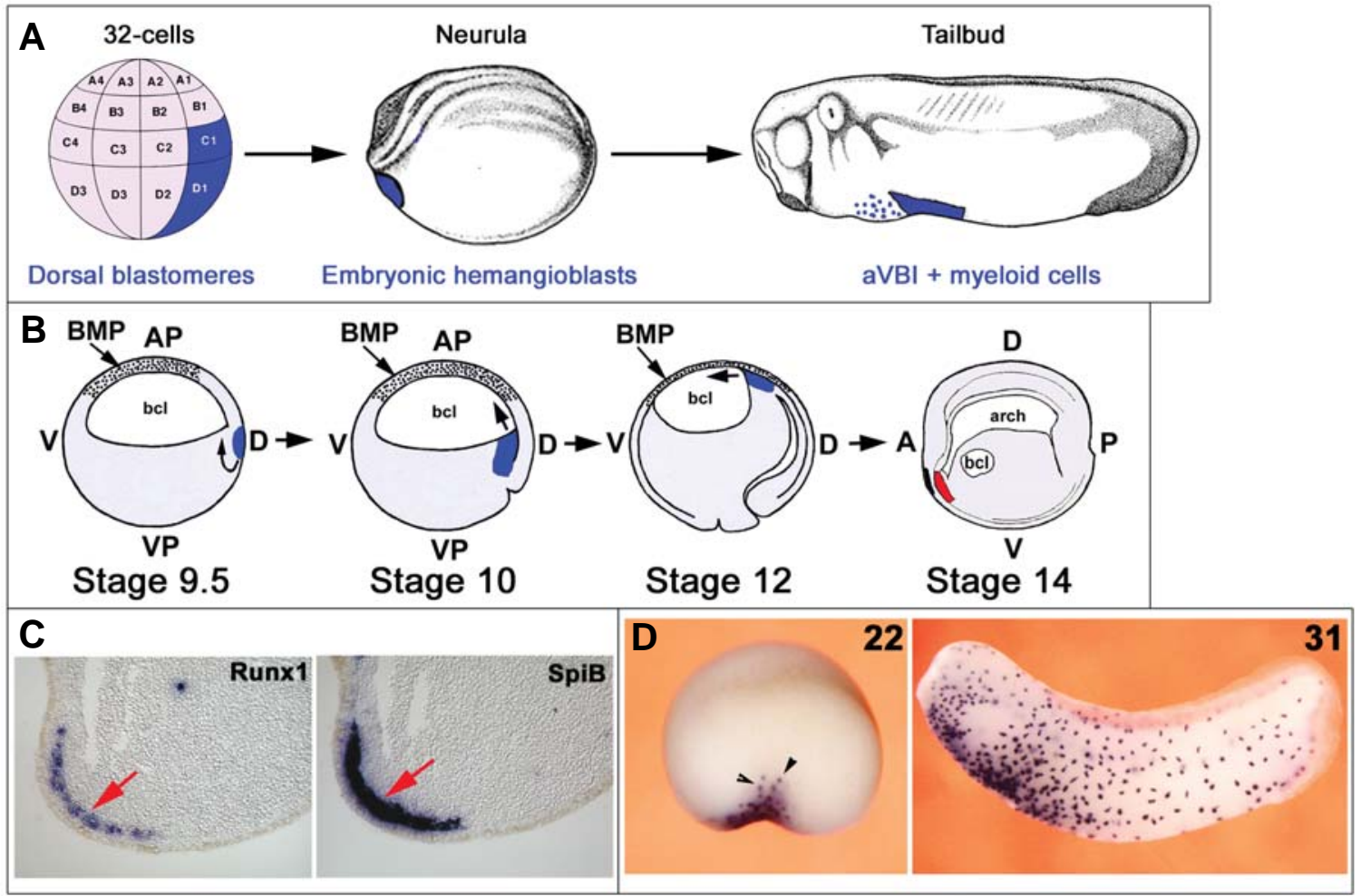

Fig. 1. Ontogeny and hematopoietic potential of the anterior portion of the ventral blood island (aVBI). (A) The aVBI and its precursor, the embryonic hemangioblasts, derive from dorsal blastomeres of the 32-cell stage embryo. (B) Embryonic hemangioblast (red cells) are specified during gastrulation when their mesodermal precursors (blue cells) encounter BMP signalling during their migration from dorsal to ventral. (C) Erythroid (Runx1) and myeloid (SpiB) genes are co-expressed (red arrows) at the hemangioblast stage. (D) Numerous myeloid cells differentiate from the embryonic hemangioblast at tail bud stages. These myeloid cells are highly migratory and initiate migration at stage 22 (24 hpf arrowheads). 
waves of hematopoiesis, the first deriving from a hemangioblast precursor and the second from hemogenic endothelium (Goldie et al., 2008; Lancrin et al., 2009). The first yolk sac wave gives rise to embryonic erythrocytes and myeloid cells whereas the second wave gives rise to multipotent progenitors and embryonic erythrocytes expressing adult globin chains (Palis, 2008). By analogy, the aVBI gives rise to embryonic erythrocytes and myeloid cells whereas the $\mathrm{pVBI}$ gives rise to multipotent progenitors differentiating into leukocytes (myeloid and a few lymphoid) in addition to embryonic erythrocytes. Thus, the aVBI appears to be equivalent to the first wave of yolk sac hematopoiesis while the pVBI is equivalent to the second wave. Whether the pVBI multipotent progenitors derive from hemogenic endothelium is currently unknown.

\section{The dorsal lateral plate (DLP)}

Studies in chimeric embryos resulted in the discovery of a second source of blood cells apart from the VBI in amphibian embryos, including Xenopus (Kau and Turpen, 1983; Maeno et al., 1985a; Turpen et al., 1981). This second source was narrowed down to the dorsal lateral plate mesoderm (DLP) (Maeno et al., 1985b), which was found to contain adult hemangioblasts that give rise to HSCs and thereby all the hematopoietic lineages of the adult (Chen and Turpen, 1995; Kau and Turpen, 1983; Turpen and Knudson, 1982; Walmsley etal., 2002). The ontogeny of adult and embryonic blood has been controversial, but a few years ago we obtained evidence in Xenopus that embryonic and adult blood cells have distinct origins (Ciau-Uitz et al., 2000). We showed that in regularly dividing embryos, the progeny of blastomere C3 includes the DLP and the HSCs associated with the ventral wall of the dorsal aorta but, critically, not the VBI (Ciau-Uitz et al., 2000; Walmsley et al., 2002; Fig. 3A and Fig. 4). Similarly, blastomeres giving rise to the VBI do not contribute to the DLP.

An alternative fate map has concluded that all $C$ and $D$ blastomeres contribute to the $\mathrm{VBI}$ and that blastomeres contributing to the DLP also contribute to the VBI and vice versa (Lane and Sheets, 2002; Lane and Smith, 1999). Reasons for this discrepancy include the use of irregularly dividing embryos and non-specific biotin staining (discussed in (Walmsley et al., 2005)). However, even under these less-than-ideal conditions the number of C3-derived cells found in the VBI was extremely low and likely to be within experimental error. Also, some of the analyses were carried out at late stages of development when the presence of highly migratory myeloid cells, and other migratory cells, could lead to spurious interpretation. As an example, Mills et al. (1999) found that at the 32-cell stage all blastomeres, including those which in other studies have been shown to exclusively give rise to ectoderm, contribute to the vasculature. This apparent disparate result could be explained by the detection of migrating neural crest cells which have been found in the vasculature and the pronephros (Collazo et al., 1993). Interestingly, though, even Mills et al., found contributions to the trunk dorsal aorta, where HSCs are found (see below), only from C3, in total agreement with our data.

Independent evidence that the DLP and VBI have different origins, comes from the ontogeny of the pronephric system. DLP hemangioblasts localise immediately dorsal to the pronephric anlagen and they co-express some pronephric genes (Walmsley et al., 2002; Fig. 3B). Hemangioblasts of the zebrafish posterior
A

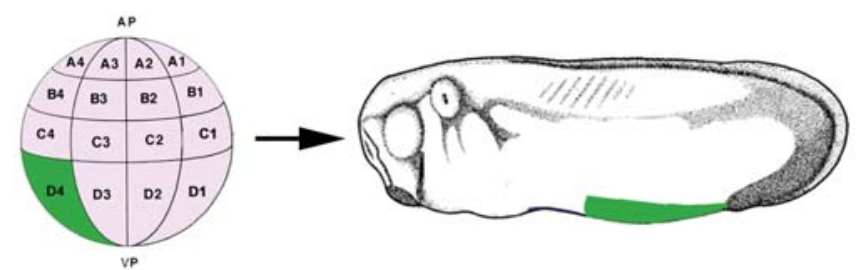

Ventral blastomere

pVBI

B

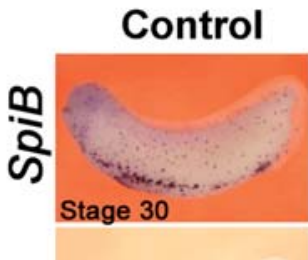

DMZ

VMZ

:
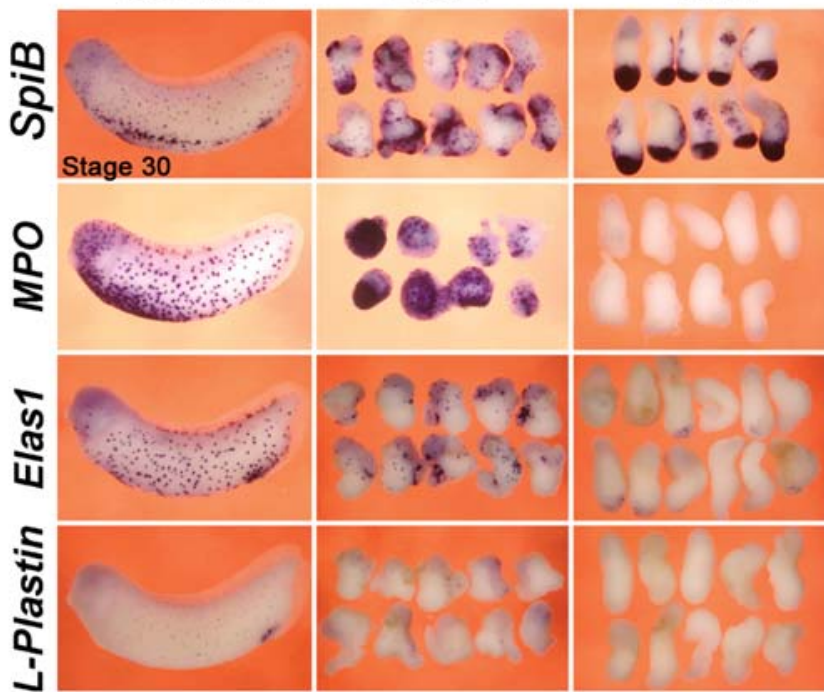

C


DMZ

VMZ

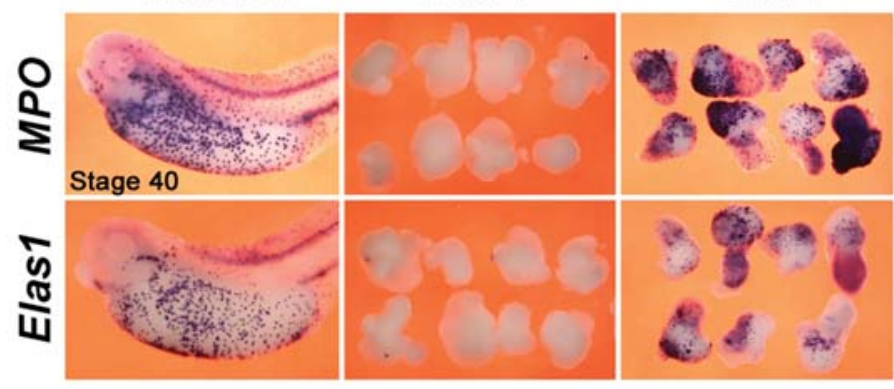

Fig. 2. Ontogeny and hematopoietic potential of the posterior portion of the ventral blood island (pVBI). (A) Ventral blastomere D4 gives rise to the pVBI. (B) At tail bud stages (stage 30, 35hpf) all differentiated myeloid cells (MPO, Elas1, L-plastin) derive from the dorsal marginal zone (DMZ) indicating their anterior hemangioblast origin. At this stage, the ventral marginal zone (VMZ) contains myeloid precursors (SpiB) but no migrating mature myeloid cells. (C) At early larval stages (stage 40,66hpf), myeloid cells are no longer produced by the DMZ while a second wave differentiating from the $V M Z$ becomes abundant.

lateral plate mesoderm (PLM) are also closely associated with pronephric tissue, and a bi-potential progenitor giving rise to pronephric cells and hemangioblasts has been proposed (Gering et al., 1998). Dale and Slack (1987) found that at the 32-cell stage the pronephric anlagen derive mainly from blastomeres $\mathrm{C} 3$, with smaller contributions from B3 and D3. Moody (1987) arrived at a similar conclusion and, in addition, pointed out that C1 and D1 do not contribute at all to pronephric tissue while D4's contribution is only small, presumably in the cloaca region. Orthotopic grafts just 
before gastrulation also indicated that the pronephric precursors are distinct from VBI precursors, and placed the pronephric progenitors (DLP) about $90^{\circ}$ from the dorsal and ventral midlines (Fig. 3C; Dale and Slack, 1987).

\section{The dorsal aorta (DA)}

Hematopoietic clusters containing HSCs are observed associated with the ventral wall of the DA at stages $42-44$ (80-92hpf) (Ciau-Uitz et al., 2000). Early transplantation experiments between cytogenetically distinct embryos demonstrated that hematopoietic cells adhering to the surface of both the DA and pronephric duct derived from DLP mesoderm (Turpen and Knudson, 1982). The DLP origin of the DA has recently been confirmed by Shibata et al. (2008), who transplanted DLPs labelled either with GFP or LacZ and found that their progeny populated the DA. Significantly, surgical elimination of the DLP results in larvae without a DA (Cleaver and Krieg, 1998). Also, the blastomere of the 32-cell stage embryo that gives rise to the DLP, namely $\mathrm{C} 3$, also gives rise to the DA of the trunk and associated intra-aortic hematopoietic clusters containing HSCs (Ciau-Uitz et al., 2000; Walmsley et al., 2002). The hypochord produces and releases diffusible isoforms of vascular endothelial growth factor (VEGF) A, which is thought to guide the migration of DA precursors from the DLP to the midline (Cleaver and Krieg, 1998). In agreement, just before the DA precursors are found coalescing ventral to the hypochord, a stream of endothelial cells are seen between the DLP and the midline (Ciau-Uitz et al., 2000).

It is important to note that hematopoiesis does not take place all along the DA but is restricted to the single medial DA encompassing the trunk, starting anteriorly just posterior to the glomus (pronephros) and ending posteriorly at the level of the cloaca (Fig. 4). A 32-cell stage fate map of the Xenopus vasculature shows that vessels which run along the anterior-posterior axis, such as the DA, have a strong anterior-posterior map (Mills et al., 1999). In other words, dorsal blastomeres give rise to the anterior portion of these vessels whereas their most posterior portion derives from ventral blastomeres. Specifically for the DA, dorsal blastomeres (A1, B1 and less significantly D1) contribute to the paired DA, which are anterior to the liver primordium and the pronephros, whereas ventral blastomeres (D4, C4 and D3) contribute to the tail artery. The trunk DA, where HSCs are found, was mapped to blastomere C3 (Mills et al., 1999; Fig. 4C). Significantly, none of the blastomeres giving rise to the $\mathrm{VBI}(\mathrm{C} 1$, D1 and D4) (Ciau-Uitz et al., 2000) contribute to the trunk $\mathrm{DA}$, and $\mathrm{C} 3$ which gives rise to the DLP and HSCs, does not contribute to the VBI. Thus, regarding the origins of the $\mathrm{VBI}$ and the DA, these two fate maps are in complete agreement and conclusively show that embryonic and adult blood have distinct origins in the Xenopus embryo.

\section{Hematopoietic development in zebrafish}

In recent years, the zebrafish has emerged as a powerful genetic model for vertebrate developmental biology. The isolation of numerous mutant lines, together with a range of other genetic perturbation approaches, has revealed that the molecular basis of hematopoiesis is highly conserved between teleosts and higher vertebrates, including humans (de Jong and Zon, 2005). Also, the generation of a handful of transgenic lines has facilitated the in vivo analysis, in real time, of developing hematopoietic cells (de Jong and Zon, 2005). As in other vertebrates, there are two major waves of hematopoiesis in zebrafish. The first wave, primitive hematopoiesis, takes place in two distinct areas of the embryo: the anterior lateral plate mesoderm (ALM) and the posterior lateral plate mesoderm (PLM, Fig. 5 A-E). At early somitogenesis stages, the ALM gives rise to a transient hemangioblast population that differentiates into myeloid and endothelial cells. In its turn, the PLM gives rise to a second population of hemangioblasts that differentiate into erythroid and endothelial cells. Red blood cells do not differentiate in the PLM, instead the erythroid derivatives of the PLM migrate to the midline to differentiate in the intermediate cell mass (Fig. 5E). Their differentiation potentials indicate that the ALM and PLM are genetically distinct, and it has been demonstrated, for example, that ALK8 signalling is essential for the ALM but dispensable for PLM specification (Hogan et al., 2006). Also, specifi-
Fig. 3. Ontogeny of dorsal lateral plate (DLP) hemangioblasts and their molecular make-up. (A) The C3 blastomere of the 32-cell stage embryo gives rise to the DLP which later generates HSCs in the ventral wall of the dorsal aorta. (B) Expression hierarchy in the DLP mesoderm. DLPhemangioblasts are located ventral to the somites and immediately dorsal to the pronephric duct and they express pronephric genes (Walmsleyet al., 2002). Pronephric genes are expressed from mid gastrula but it is not until early tail bud (Stage 20,20hpf) that endothelial expression is seen in a subset of pronephric cells. A few hours later, endothelial expressing cells initiate hematopoietic expression to become hemangioblasts. (C) Orthotopic grafts at the beginning of gastrulation (stage 10,11hpf) indicate that the region giving rise to the pronephros and the DLP is located $90^{\circ}$ from both the $D M Z$ and $V M Z$, illustrating the distinct origins of the VBI and DLP. 
A

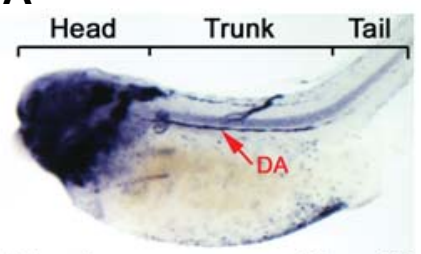

B

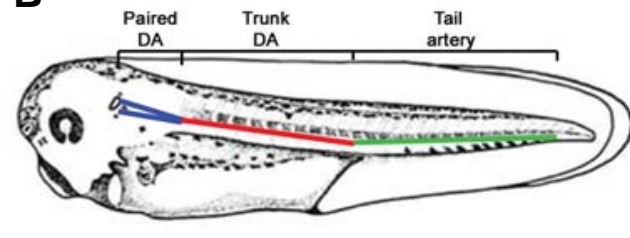

Runx1

C

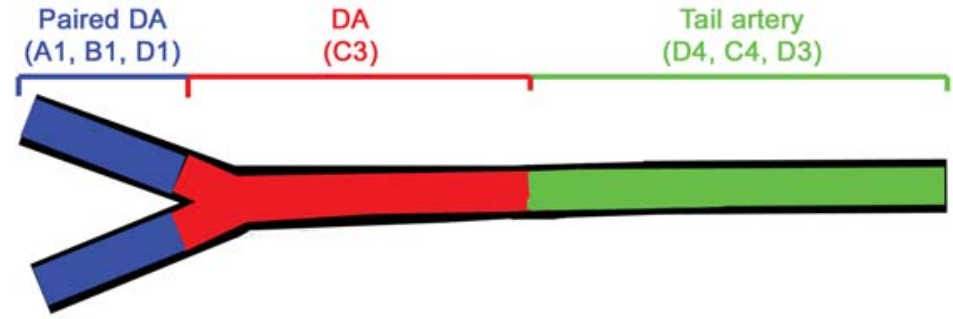

Fig. 4. Ontogeny of the dorsal aorta (DA) and hematopoietic stem cells (HSCs) in Xenopus. (A) Runx 1 expression and HSC emergence is restricted to the DA encompassing the trunk. (B) The DA is anatomically subdivided into paired $D A$, trunk DA and tail artery. HSCs only emerge in the trunk DA. (C) Diagram summarizing the blastomere origins of the anatomical subdivision of the DA.

cation of the ALM appears to be intimately interconnected with the development of cardiac tissue: the elimination of the ALM programme causing an expansion of the heart (Schoenebeck et al., 2007).

The second wave of hematopoiesis, definitive hematopoiesis, gives rise to HSCs, which generate all blood cell types in the later embryo and throughout adult life. In the adult, the head kidney serves as the main hematopoietic organ (Willett et al., 1999), and its function is equivalent to that of the bone marrow in mammals. The derivatives of the HSCs include erythroid (erythrocyte), myeloid (macrophage, neutrophil, monocyte) and lymphoid (B and $\mathrm{T}$ cell). Apart from megakaryocytes and natural killer (NK) cells, all other differentiated blood lineages have been found and characterized in zebrafish embryos and/or adults, and they exhibit a high degree of conservation in both molecular and cellular features when compared to their counterparts in mammals.

Recent lineage tracing has revealed the migratory pathways of HSCs in zebrafish embryos. As in mammals, the first HSCs are found associated with the ventral wall of the DA. At least partly via the circulation, these HSCs migrate and colonize a recently discovered hematopoietic organ, the caudal hematopoietic tissue (CHT), which is believed to be functionally equivalent to the mammalian fetal liver (Murayama et al., 2006). HSCs expand in the CHT and some of them differentiate into erythrocytes and myeloid cells while others further migrate to the thymus and the kidney, the main hematopoietic organ in adult fish (Jin et al., 2007; Murayama et al., 2006). In addition, recent work provides evidence for a novel migration route in which DA-derived CD41+cmyb+ cells colonize the zebrafish kidney by migrating along the pronephric tubules (Bertrand et al., 2008). The pancreas is the site for B cell differentiation in zebrafish (Danilova and Steiner, 2002), however the origins of the precursors and their migratory routes are not known.

\section{Cellular intermediates and signalling}

\section{The hemangioblast}

During development, blood and endothelial cells develop in close proximity to each other suggesting that they derive from a shared common progenitor cell, termed the hemangioblast (Sabin, 1920). Consistent with such a notion, many hematopoietic and endothelial genes are co-expressed during development, and deletion of some of them causes both blood and endothelial defects. The first cellular evidence for hemangioblasts was provided by studies of embryonic stem (ES) cells, where individual cultured cells give rise to colonies containing both hematopoietic and endothelial cells (Choi et al., 1998). Although this

Fig. 5. Origins of hemangioblast and blood/endothelial cells in zebrafish embryos. (A,B) Expression patterns of

flk1 (blue) and gata1 (red) in a flat mounted 8-somite stage embryo, with myoD marking the somites. (A) Schematic diagram; (B) in situ hybridization. (C-E) Expression pattern of an established hemangioblast marker, scl, in zebrafish marks both the anterior hemangioblast population (ALM) which will give rise to myeloid and endothelial cells, and the posterior hemangioblast population (PLM) which will give rise to erythroid and endothelial cells. $(\mathbf{F}, \mathbf{G})$ Lineage tracing shows that PLM cells labeled at $5 s(\mathbf{F})$ by photo conversion of fluorescent protein (Hatta et al., 2006) in the confocal microscope, are found migrating to the midline at $18 \mathrm{~s}$ (not shown) and differentiated into red blood cells and endothelial cells at 26 hpf (G), with arrow indicating endothelial cells. Flat mount view (A-D); lateral view (E-G).

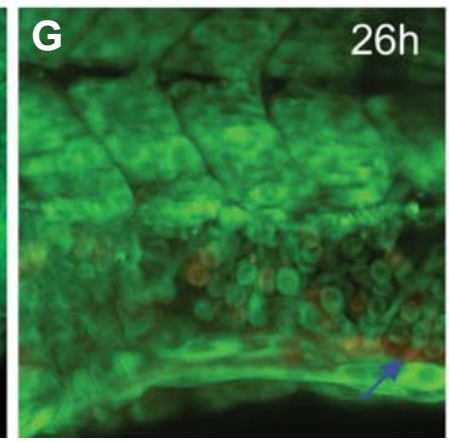

E $26 \mathrm{~h}$ primitive blood scl

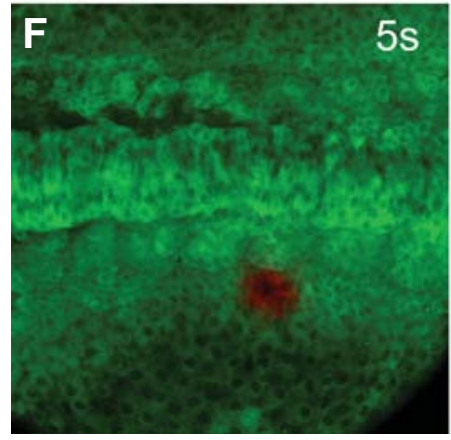

D
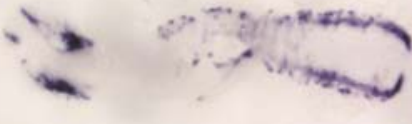

$6 h$ 
shows that blood and endothelium can derive from a common progenitor, the number of such bipotential progenitor cells in vivo appears to be too low to account for all the blood and endothelium.

In the mouse, single cells isolated from the posterior primitive streak give rise to blood and endothelial cells, indicating that hemangioblasts are generated during gastrulation (Huber et al., 2004). Similarly, single cell labelling and lineage tracing experiments in zebrafish identified cells with hematopoietic and endothelial potential in the gastrulating embryo (Vogeli et al., 2006). These experiments indicate that bipotential progenitors are located in the ventral blastomeres at shield stage, however the low numbers of such cells suggest that only a subset of hematopoietic and endothelial cells arise from hemangioblasts. Labelling cells during gastrulation could target mesodermal cells with a broader potential and give rise to many mesoderm-derived tissues. To explore the potential of more committed cells, we labelled cells at the early somitogenesis stage when hemangioblast populations are defined by the co-expression of genes such as $s c /$, flit and gata2(Fig. 5 F-G). Using a photo convertible fluorescent protein (green to red), a group of posterior lateral plate (PLM) cells were labeled under the confocal microscope at 5s (Fig. 5F). Time lapse imaging shows that these cells migrated to the midline and eventually differentiated into blood and endothelial cells at $26 \mathrm{hpf}$ (Fig. 5G). Thus, clearly the PLM contains precursors of both blood and endothelium at early somitogenesis stages (as already shown by Vogeli et al., 2006; Zhang and Rodaway, 2007). However, the existence of 'hemangioblasts' in the PLM awaits the labeling of single cells within this population.

In mouse embryos many endothelial cells of the yolk sac arise from unilineage angioblast precursors and not from hemangioblasts (Furuta et al., 2006; Ueno and Weissman, 2006). Furthermore, even though all hematopoietic cells, and some endothelial cells, had been thought to arise from hemangioblast precursors (Palis, 2008), recent lineage tracing has questioned this (Ueno and Weissman, 2006). In zebrafish embryos, most of the blood and the endothelium appears to come from cells which earlier express $s c l$, a criterion used in some mouse circles as a definition of the hemangioblast (Chung et al., 2002; Gering et al., 1998). In the frog, while many of the endothelial cells derived from the anterior ventral blood island mesoderm, the vitelline vessels, derive from $s c /$ positive cells, those in the posterior and in the head appear not to go through an sc/expressing stage. We therefore conclude that the extent to which individual vertebrate species make blood and endothelium from a hemangioblast precursor may vary. Regardless of whether it goes through a hemangioblast intermediate or not, all blood and endothelial development appears to require bmp signaling (Walmsley et al., 2002).

\section{Hemogenic endothelium}

Emergence of the first HSCs is intimately associated with the ventral wall of the DA and, in mammals, the vitelline and umbilical arteries (Ciau-Uitz etal., 2000; Gering and Patient, 2005; Jaffredo et al., 1998; Wang et al., 1998). A body of evidence indicates that HSCs derive from cells with an endothelial phenotype and suggests that HSCs differentiate from the endothelium, hemogenic endothelium, of mature blood vessels (Chen et al., 2009; Taoudi et al., 2008; Zovein et al., 2008). Interestingly, HSC emergence has only been associated with arteries (de Bruijn et al., 2000). Arteries and veins are functionally and structurally distinct and recent reports indicate that their genetic programming is also different. Studies from zebrafish and other vertebrates demonstrate that a complex network of molecular pathways is involved in the specification of veins and arteries during embryogenesis (Gering and Patient, 2005; Lawson et al., 2001; Lawson et al., 2002; Wang et al., 1998). Genetic mutants combined with other manipulations including chemical inhibitors have clearly demonstrated that a hedgehog $(\mathrm{HH})$-VEGF-Notch pathway is required for arterial specification of the DA. In this signalling cascade, $\mathrm{HH}$ expressed in the notochord, hypochord and floor plate, induces VEGF expression in nearby somites and, in its turn, VEGF activates Notch in the DA to induce arterial differentiation in part by suppressing venous fate (Lawson et al., 2002). How VEGF regulates Notch is currently unclear. Nevertheless, recent work in the mouse shows that VEGF activates ERK signaling to induce expression of DII4 and Hey via Foxc1/2, transcription factors (TFs) that directly bind to the Dll4 promoter (Hayashi and Kume, 2008; Seo et al., 2006). Furthermore, the amount of exogenous VEGF added to cultured endothelial cells greatly influences their differentiation into veins or arteries, with lower levels of VEGF favouring vein differentiation and higher levels inducing arterial specification (Lanner et al., 2007). This indicates that VEGF availability is a key factor during arterial specification. Strikingly, a HH-VEGF-Notch signalling cascade is also required for the emergence of HSCs in the ventral wall of the DA (Gering and Patient, 2005). However, how exactly this cascade operates to control HSC emergence is currently unknown and whether arterial specification is a prerequisite for HSC emergence or a parallel developmental process is not known. However, recent data from our lab shows that signalling by bmp4 from underneath the DA is required to induce the HSC programme in the floor of the DA (Wilkinson et al., 2009). In its absence, the arterial programme is intact, consistent with the increasing evidence that an arterial endothelial cell is the immediate precursor to the HSC, and with the conversion from one to the other being driven by BMP signaling. Distance of the DA floor from continued $\mathrm{HH}$ signaling also appears to be a factor (Wilkinson et al., 2009).

Hemogenic endothelium has also been described in the mouse yolk sac (Goldie et al., 2008) and in cultured ES cells (Lancrin et al., 2009). Clonal analyses indicate that Flk1 ${ }^{+} \mathrm{C}-\mathrm{Kit}^{+} \mathrm{CD} 45^{-}$side population cells isolated from the murine yolk sac represent hemogenic endothelium with multi lineage hematopoietic potential. This rare cell population constitutes approximately $0.05-0.1 \%$ of yolk sac cells and localizes to the yolk sac capillaries, particularly on the arterial side of the yolk sac vasculature, but also to the vitelline vein, with the greatest concentration in the latter (Goldie et al., 2008). Since hemogenic endothelium is thought to be associated to arteries rather than to veins, the presence of $\mathrm{Flk} 1^{+} \mathrm{C}-$ $\mathrm{Kit}^{+} \mathrm{CD} 45^{-}$cells in the vitelline vein is intriguing and further clarification of their hematopoietic potential is required. Interestingly, yolk sac hemogenic endothelium expresses retinoic acid receptors and, in contrast to the first wave of yolk sac hematopoiesis, their hematopoietic differentiation is dependent on retinoic acid signalling (Goldie et al., 2008). More recently, Tie $2^{\text {hi }} \mathrm{c}$ $\mathrm{Kit}^{+} \mathrm{CD} 41^{-}$cells isolated from ES cells and in gastrulating murine embryos have been shown to represent hemogenic endothelium (Lancrin et al., 2009). These cells are located in developing yolk sac blood islands of early head-fold embryos and have the capacity to produce both primitive red cells and pro-definitive 
hematopoietic colonies giving rise to red cells expressing adult hemoglobin isoforms and myeloid cells. Hierarchically, hemogenic endothelium may represent an intermediate between hemangioblasts, found in the primitive streak and from which they arise, and hematopoietic precursors found in the yolk sac (Lancrin et al., 2009).

\section{Transcriptional control}

Hematopoietic cell fate decisions are ultimately controlled by transcription factors (TFs) regulating gene expression. A substantial number of TFs have been implicated in hematopoietic development. Amongst them, runx 1 has been shown to be essential for HSC emergence in vertebrates, including Xenopus and zebrafish (Burns et al., 2005; Gering and Patient, 2005; KalevZylinska et al., 2002; Our unpublished data). Other key hematopoietic TFs, such as scl, Imo2and gata2, affect both primitive and definitive hematopoiesis. These TFs play multiple roles in hematopoiesis making the elucidation of these roles in hemangioblast and HSC emergence, for example, dependent on the development of new tools with stage and lineage specificity. Here we

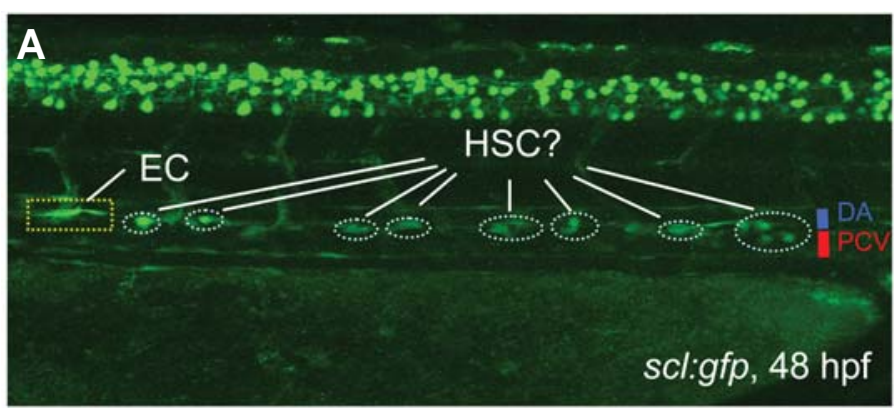

B

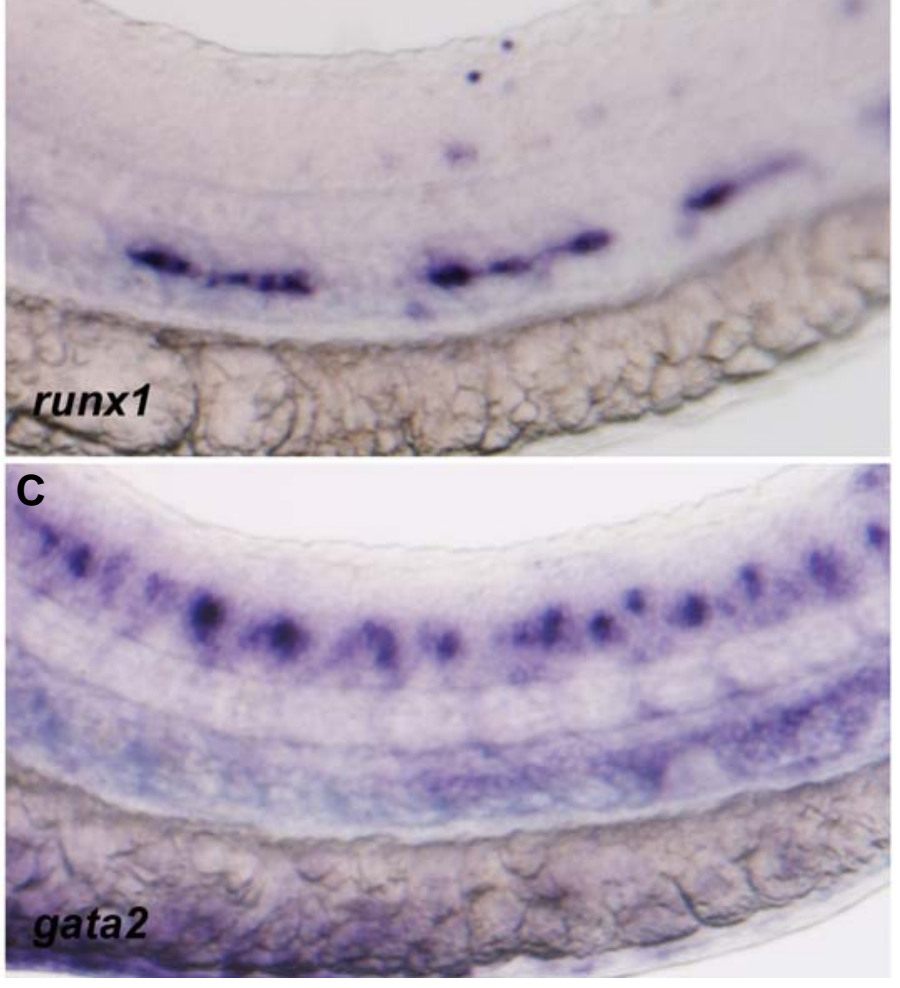

summarize the current knowledge of the functions of key TFs in hematopoietic development, although we start with a still-mysterious player, cloche.

\section{Cloche}

In zebrafish, the cloche mutant is the earliest mutant identified so far with both hematopoietic and endothelial lineage defects (Stainier et al., 1995). The recently cloned mouse homologue of a gene cloned from the cloche interval, lysocardiolipin acyltransferase (lycat), has been shown to be essential for the development of hematopoietic and endothelial lineages during in vitro ES cell differentiation (BL-CFC assay) (Wang et al., 2007). Interestingly, protein modification by acyltransferase has emerged as an important mechanism for regulating cytoplasmic and secreted signalling molecules, such as Hedgehogs and Wnts (Linder and Deschenes, 2003; Linder and Deschenes, 2004; Wang et al., 2007). This suggests that Lycat could modulate the secretion and extracellular availability of molecules required for hematopoiesis, for example, HHs, Wnts, Bmps, VEGF etc. Alternatively, Lycat could regulate the nuclear availability of TFs such as Scl, Runx1 and others to control the specification of the hematopoietic and endothelial lineages (Chamoun et al., 2001; Lee and Treisman, 2001; Wang et al., 2007).

\section{Scl/Lmo2}

The $\mathrm{T}$ cell leukemia oncoprotein, $\mathrm{Scl} / \mathrm{Tal}-1$, is a basic-helixloop-helix TF, required for production of embryonic red blood cells in the mouse yolk sac (Shivdasani et al., 1995). In zebrafish, Scl plays early and pivotal roles during the specification of both blood and endothelial cells, possibly at the hemangioblast level (Dooley et al., 2005; Gering et al., 1998; Gering et al., 2003; Patterson et al., 2005). Ectopic expression of Scl mRNA in zebrafish embryos results in overproduction of hemangioblasts (Gering et al., 1998). Morpholino injections showed that $\mathrm{Scl}$ is required for both primitive and definitive hematopoiesis and, in addition, for DA formation, which may explain the lack of HSCs in $s c /$ morphants (Patterson et al., 2005). To better understand the development of $s c /+$ cells in zebrafish, we generated an scl:GFPtransgenic line. The location of scl:GFPcells in the DA (Fig. 6A) is similar to those expressing runX1, a well established marker for definitive HSCs in vertebrates (Fig. 6B), indicating that, in addition to hemangioblasts, HSC clusters in zebrafish express high levels of SCL.

Lmo2encodes a cysteine-rich, two LIM-domain protein that is required for yolk sac erythropoiesis (Warren et al., 1994). The Lmo2 protein has a central and crucial role in hematopoietic development and is highly conserved. However, its role in vasculogenesis was only identified very recently in zebrafish. Lmo2-deficient embryos show a phenotype similar to $s c /$ morphants, that is, a lack of primitive and definitive blood and deficient DA development (Patterson et al., 2007; Patterson etal., 2005). Simultaneous overexpression of Scl and Lmo2 converts non-axial mesoderm into hemangioblasts which differentiate into

Fig. 6. HSCs in association with the ventral wall of the dorsal aorta (DA). (A) scl:gfp transgenic embryos show GFP expression in the putative HSCs in the DA at 48 hpf. (B) In situ hybridization at 26hpf showing runx1 expressing cells (HSCs) associated with the ventral wall of the DA. (C) gata2 is expressed in primitive blood, vessels and spinal chord neurons at $26 \mathrm{hpf}$. Lateral views, anterior to the left. 
endothelial cells in the absence of Gata1 (Gering et al., 2003), further supporting a role for an Scl-Lmo2 complex in the specification of hemangioblasts. Importantly, rescue of $\mathrm{sc} /$ morphants only occurs if the rescuing Scl can interact with Lmo2 (Patterson et al., 2007).

\section{Gata2}

Gata-2 is an essential TF in the hematopoietic system, including hematopoietic stem and progenitor cells. Mice lacking Gata2 suffer from severely impaired primitive erythropoiesis and a complete lack of HSCs and other committed progenitors (Tsai et al., 1994). In zebrafish, gata2 is expressed predominantly in primitive blood and weakly in vessels (Fig. 6C). However, no convincing demonstration of an early and critical role for zebrafish Gata2 has been provided (Galloway et al., 2008). In contrast, studies in Xenopus embryos clearly demonstrate that Gata2 is required for hemangioblast formation by controlling several important regulators (such as Scl and Lmo2) but not Fli1 (Liu et al., 2008).

\section{ETS transcription factors: Fli1 and Etsrp}

Genes necessary for blood and endothelial development contain ETS binding sites in their promoters/enhancers, suggesting that ETS factors act at the top of the transcriptional network driving blood and endothelial development via direct regulation of several master regulators. Their precise roles in this network were evaluated in manipulated zebrafish and Xenopusembryos, where the populations of blood and endothelial precursors are welldefined (Liu et al., 2008). By gain- and loss-of-function analyses we showed that Fli1 is both necessary and sufficient for hemangioblast formation in vivo. It works by inducing expression of key regulators of hematopoiesis and vasculogenesis, namely Scl, Lmo2, Gata2, Etsrp and Flk1, and it also positively feeds back on its own expression. The misprogramming of the hemangioblast population which takes place in the absence of Fli1 is followed by apoptosis. Epistatic experiments show that, although induction of Fli1 expression is not dependent on the activities of Scl, Gata2, Etsrp or Cloche, its activation of downstream target genes does depend on the activities of these important regulators, indicating a position either upstream of or parallel to them in the genetic hierarchy leading to blood and endothelial development. Taken together, these results identify Fli1, as a master regulator of the hemangioblast programme in vertebrate embryos.

Etsrp, a novel zebrafish ETS domain protein, has been shown to be necessary and sufficient for the initiation of vasculogenesis in zebrafish (Sumanas and Lin, 2006). However, recent observations by us and others have suggested that Etsrp may play an earlier role during hemangioblast formation in the ALM, since loss of Etsrp causes an absence of both myeloid and endothelial makers in the ALM (Liu and Patient, 2008; Sumanas et al., 2008). This view is supported by functional analysis of the mouse homolog of Etsrp, ER71/ETV2 (Lee et al., 2008). ER71 enforced expression in ES cells appears to be sufficient to induce the generation of Flk $1^{+}$cells and enhance hematopoietic and endothelial cell generation even when BMP, Notch and Wnt are inhibited. Furthermore, in ER71\% embryos a complete absence of $\mathrm{Flk} 1^{+}$hematopoietic and endothelial cells is observed (Lee et al., 2008), indicating that ER71 is essential for the generation of Flk1+ mesoderm and its derivatives.

\section{A genetic regulatory network for hematopoietic develop- ment}

The specification of hemangioblasts from mesoderm and their differentiation into blood and endothelial cells requires precise coordination of TF activity. A fuller understanding of development will require moving beyond the study of individual genes to determine how regulatory genes interact to form functional genetic regulatory networks (GRNs) (Levine and Davidson, 2005). Conservation of the vertebrate body plan has been attributed to the evolutionary stability of such GRNs, which consist of assemblies of subcircuits made up of TFs and their target cis-regulatory modules. Subcircuits that perform essential functions in building up body parts have been termed kernels of the GRNs (Davidson and Erwin, 2006). Disruption of TFs that make up the kernel often results in loss of the body part, and hence the basic architecture of the kernel is highly conserved through evolution (Davidson and Erwin, 2006).

In vitro data suggests that the $s c / g e n e$ may be regulated by a TF complex that consists of the ETS transcription factors, Fli1 and Elf1, and Gata2 (Gottgens et al., 2002). Indeed, recent data suggests that fli1, sc/and gata2 may reciprocally regulate each other in hematopoietic progenitor and endothelial cell lines, possibly also in hematopoietic tissues (i.e. fetal liver and the AGM) and in BL-CFC, the in vitroequivalent of hemangioblasts (Pimanda et al., 2007). It has been suggested that flit, sc/and gata2form an auto-regulatory feedback loop (a GRN kernel; Fig. 7) that regulates hemangioblast development and that this putative GRN kernel could well be conserved from invertebrates, like Drosophila, to vertebrates. Whether this GRN kernel is operational in vivo during hemangioblast specification in fish and frog embryos has recently been investigated (Liu et al., 2008). These data, suggest that Fli1 acts at the top of the genetic regulatory hierarchy controlling hemangioblast specification, since it controls the expression of several master regulators, such as scl, gata2, Imo2 and fli1 itself (Fig. 7) (Liu et al., 2008). It is likely that upstream signals, such as Bmp initiate flit and gata2expression (Walmsley

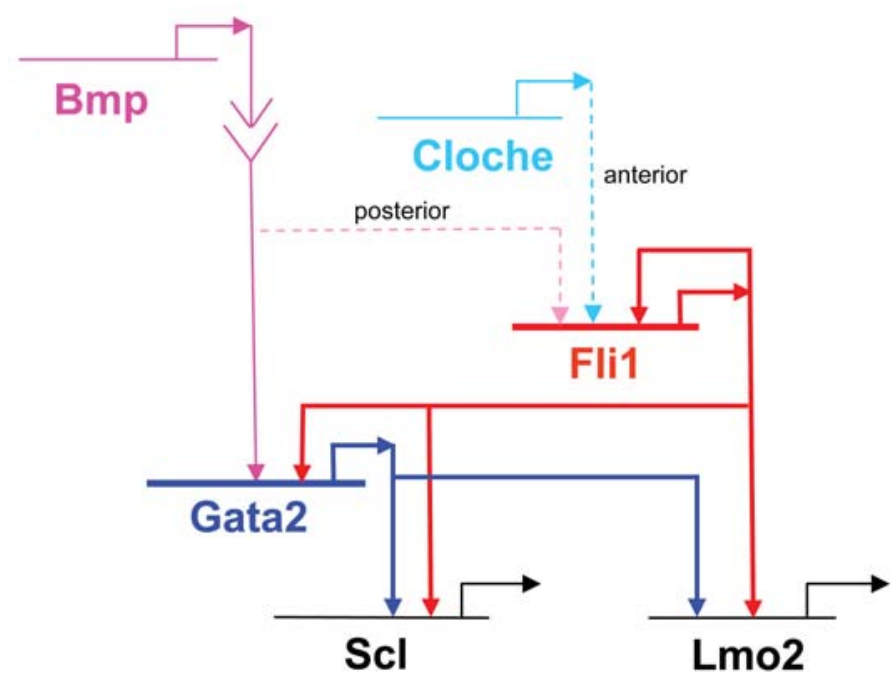

Fig. 7. The hemangioblast kernel in vertebrate embryos. Solid lines, direct regulation; dashed lines, indirect or no evidence for direct regulation; chevron connection, signalling pathway. Adapted from Liu et al., 2008. 
et al., 2002), which then autoregulate and combine to initiate $s c /$ and Imo2 expression, and then induce downstream targets. Autoregulation such as that seen for flit can sustain the network after inducing signals have ceased. Steady-state kinetics of individual TFs, threshold concentrations at which they trigger expression and the requirement for combinatorial binding could all be critical factors in this circuit to either maintain the hemangioblast state or drive differentiation (Pimanda et al., 2007).

\section{Perspectives}

Over the past few years, we have gained a better understanding of hematopoietic development in both Xenopusand zebrafish, from the ontogeny of hematopoietic sites to the details of regulatory signalling pathways and transcription factor networks. Most of the information is applicable to mammals including humans, so it should benefit attempts to make these cells in vitroand manipulate them in vivo.

Acknowledgements

This work was supported by the MRC.

\section{References}

BERTRAND, J. Y., KIM, A. D., TENG, S. and TRAVER, D. (2008). CD41+ cmyb+ precursors colonize the zebrafish pronephros by a novel migration route to initiate adult hematopoiesis. Development 135: 1853-1862.

BURNS, C. E., TRAVER, D., MAYHALL, E., SHEPARD, J. L. and ZON, L. I. (2005). Hematopoietic stem cell fate is established by the Notch-Runx pathway. Genes Dev 19: 2331-2342.

CHAMOUN, Z., MANN, R. K., NELLEN, D., VON KESSLER, D. P., BELLOTTO, M., BEACHY, P. A. and BASLER, K. (2001). Skinny hedgehog, an acyltransferase required for palmitoylation and activity of the hedgehog signal. Science 293: 2080-2084

CHEN, M. J., YOKOMIZO, T., ZEIGLER, B. M., DZIERZAK, E. and SPECK, N. A. (2009). Runx1 is required for the endothelial to haematopoietic cell transition but not thereafter. Nature 457: 887-891.

CHEN, X. D. and TURPEN, J. B. (1995). Intraembryonic origin of hepatic hematopoiesis in Xenopus laevis. $J$ Immuno/ 154: 2557-2567.

CHOI, K., KENNEDY, M., KAZAROV, A., PAPADIMITRIOU, J. C. and KELLER, G. (1998). A common precursor for hematopoietic and endothelial cells. Development 125: 725-732.

CHUNG, Y. S., ZHANG, W. J., ARENTSON, E., KINGSLEY, P. D., PALIS, J. and $\mathrm{CHOI}, \mathrm{K}$. (2002). Lineage analysis of the hemangioblast as defined by FLK1 and SCL expression. Development 129: 5511-5520.

CIAU-UITZ, A., WALMSLEY, M. AND PATIENT, R. (2000). Distinct origins of adult and embryonic blood in Xenopus. Cel/102: 787-796.

CLEAVER, O. and KRIEG, P. A. (1998). VEGF mediates angioblast migration during development of the dorsal aorta in Xenopus. Development 125: 39053914.

COLLAZO, A., BRONNER-FRASER, M. and FRASER, S. E. (1993). Vital dye labelling of Xenopus laevis trunk neural crest reveals multipotency and novel pathways of migration. Development 118: 363-376.

COSTA, R. M., SOTO, X., CHEN, Y., ZORN, A. M. and AMAYA, E. (2008). spib is required for primitive myeloid development in Xenopus. Blood112, 2287-2296.

DALE, L. and SLACK, J. M. (1987). Fate map for the 32-cell stage of Xenopus laevis. Development 99: 527-551.

DANILOVA, N. and STEINER, L. A. (2002). B cells develop in the zebrafish pancreas. Proc Natl Acad Sci USA 99: 13711-13716.

DAVIDSON, E. H. and ERWIN, D. H. (2006). Gene regulatory networks and the evolution of animal body plans. Science 311 : 796-800.
DE BRUIJN, M. F., SPECK, N. A., PEETERS, M. C. and DZIERZAK, E. (2000). Definitive hematopoietic stem cells first develop within the major arterial regions of the mouse embryo. EMBO J19: 2465-2474.

DE JONG, J. L. and ZON, L. I. (2005). Use of the zebrafish system to study primitive and definitive hematopoiesis. Annu Rev Genet 39: 481-501.

DOOLEY, K. A., DAVIDSON, A. J. and ZON, L. I. (2005). Zebrafish scl functions independently in hematopoietic and endothelial development. Dev Bio/277: 522-536.

DUPASQUIER, L., ROBERT, J., COURTET, M. and MUSSMANN, R. (2000). B-cell development in the amphibian Xenopus. Immunol Rev 175: 201-213.

FURUTA, C., EMA, H., TAKAYANAGI, S., OGAERI, T., OKAMURA, D., MATSUI, Y. and NAKAUCHI, H. (2006). Discordant developmental waves of angioblasts and hemangioblasts in the early gastrulating mouse embryo. Development 133 2771-2779.

GALLOWAY, J. L., WINGERT, R. A., THISSE, C., THISSE, B. and ZON, L. I. (2008). Combinatorial regulation of novel erythroid gene expression in zebrafish. Exp Hemato/36: 424-432.

GERING, M. and PATIENT, R. (2005). Hedgehog signaling is required for adult blood stem cell formation in zebrafish embryos. Dev Cel/8: 389-400.

GERING, M., RODAWAY, A. R., GOTTGENS, B., PATIENT, R. K. and GREEN, A R. (1998). The SCL gene specifies haemangioblast development from early mesoderm. EMBO J17: 4029-4045.

GERING, M., YAMADA, Y., RABBITTS, T. H. and PATIENT, R. K. (2003). Lmo2 and $\mathrm{Scl} /$ Tal1 convert non-axial mesoderm into haemangioblasts which differentiate into endothelial cells in the absence of Gata1. Development 130: 6187-6199.

GOLDIE, L. C., LUCITTI, J. L., DICKINSON, M. E. and HIRSCHI, K. K. (2008). Cell signaling directing the formation and function of hemogenic endothelium during murine embryogenesis. Blood 112: 3194-3204.

GOTTGENS, B., NASTOS, A., KINSTON, S., PILTZ, S., DELABESSE, E. C., STANLEY, M., SANCHEZ, M. J., CIAU-UITZ, A., PATIENT, R. and GREEN, A. R. (2002). Establishing the transcriptional programme for blood: the SCL stem cell enhancer is regulated by a multiprotein complex containing Ets and GATA factors. EMBO J21: 3039-3050

HATTA, K., TSUJII, H. and OMURA, T. (2006). Cell tracking using a photoconvertible fluorescent protein. Nat Protoc 1: 960-7.

HAYASHI, H. and KUME, T. (2008). Foxc transcription factors directly regulate DII4 and Hey2 expression by interacting with the VEGF-Notch signaling pathways in endothelial cells. PLOS One 3: e2401.

HOGAN, B. M., LAYTON, J. E., PYATI, U. J., NUTT, S. L., HAYMAN, J. W., VARMA, S., HEATH, J. K., KIMELMAN, D. and LIESCHKE, G. J. (2006). Specification of the primitive myeloid precursor pool requires signaling through Alk8 in zebrafish. Curr Biol 16: 506-511.

HUBER, T. L., KOUSKOFF, V., FEHLING, H. J., PALIS, J. and KELLER, G. (2004). Haemangioblast commitment is initiated in the primitive streak of the mouse embryo. Nature 432: 625-630.

JAFFREDO, T., GAUTIER, R., EICHMANN, A. and DIETERLEN-LIEVRE, F (1998). Intraaortic hemopoietic cells are derived from endothelial cells during ontogeny. Development 125: 4575-4583.

JIN, H., XU, J. and WEN, Z. (2007). Migratory path of definitive hematopoietic stem/ progenitor cells during zebrafish development. Blood 109: 5208-5214.

KALEV-ZYLINSKA, M. L., HORSFIELD, J. A., FLORES, M. V., POSTLETHWAIT, J. H., VITAS, M. R., BAAS, A. M., CROSIER, P. S. and CROSIER, K. E. (2002). Runx1 is required for zebrafish blood and vessel development and expression of a human RUNX1-CBF2T1 transgene advances a model for studies of leukemogenesis. Development 129; 2015-2030.

KAU, C. L. and TURPEN, J. B. (1983). Dual contribution of embryonic ventral blood island and dorsal lateral plate mesoderm during ontogeny of hemopoietic cells in Xenopus laevis. J Immuno/ 131: 2262-2266.

KUMANO, G., BELLUZZI, L. and SMITH, W. C. (1999). Spatial and temporal properties of ventral blood island induction in Xenopus laevis. Development 126: 5327-5237.

LANCRIN, C., SROCZYNSKA, P., STEPHENSON, C., ALLEN, T., KOUSKOFF, V. and LACAUD, G. (2009). The haemangioblast generates haematopoietic cells through a haemogenic endothelium stage. Nature 457: 892-895.

LANE, M. C. and SHEETS, M. D. (2002). Primitive and definitive blood share a 


\section{A. Ciau-Uitz et al.}

common origin in Xenopus: a comparison of lineage techniques used to construct fate maps. Dev Bio/248: 52-67.

LANE, M. C. and SMITH, W. C. (1999). The origins of primitive blood in Xenopus: implications for axial patterning. Development 126; 423-434.

LANNER, F., SOHL, M. and FARNEBO, F. (2007). Functional arterial and venous fate is determined by graded VEGF signaling and notch status during embryonic stem cell differentiation. Arterioscler Thromb Vasc Bio/27: 487-493.

LAWSON, N. D., SCHEER, N., PHAM, V. N., KIM, C. H., CHITNIS, A. B., CAMPOSORTEGA, J. A. and WEINSTEIN, B. M. (2001). Notch signaling is required for arterial-venous differentiation during embryonic vascular development. Deve/opment 128: 3675-3683.

LAWSON, N. D., VOGEL, A. M. and WEINSTEIN, B. M. (2002). sonic hedgehog and vascular endothelial growth factor act upstream of the Notch pathway during arterial endothelial differentiation. Dev Ce//3; 127-136.

LEE, D., PARK, C., LEE, H., LUGUS, J. J., KIM, S. H., ARENTSON, E., CHUNG, Y. S., GOMEZ, G., KYBA, M., LIN, S., JANKNECHT, R., LIM, D. S. and CHOI, K. (2008). ER71 acts downstream of BMP, Notch, and Wnt signaling in blood and vessel progenitor specification. Cel/ Stem Cel/2: 497-507.

LEE, J. D. and TREISMAN, J. E. (2001). Sightless has homology to transmembrane acyltransferases and is required to generate active Hedgehog protein. Curr Bio/ $11 ; 1147-1152$

LEVINE, M. and DAVIDSON, E. H. (2005). Gene regulatory networks for development. Proc Natl Acad Sci USA 102: 4936-4942.

LINDER, M. E. and DESCHENES, R. J. (2003). New insights into the mechanisms of protein palmitoylation. Biochemistry 42; 4311-4320.

LINDER, M. E. and DESCHENES, R. J. (2004). Model organisms lead the way to protein palmitoyltransferases. J Cel/ Sci117: 521-526.

LIU, F. and PATIENT, R. (2008). Genome-Wide Analysis of the Zebrafish ETS Family Identifies Three Genes Required for Hemangioblast Differentiation or Angiogenesis. Circ Res 103: 1147-1154.

LIU, F., WALMSLEY, M., RODAWAY, A. and PATIENT, R. (2008). Fli1 acts at the top of the transcriptional network driving blood and endothelial development. Curr Biol18: 1234-1240.

MAENO, M., TOCHINAI, S. and KATAGIRI, C. (1985a). Differential participation of ventral and dorsolateral mesoderms in the hemopoiesis of Xenopus, as revealed in diploid-triploid or interspecific chimeras. Dev Bio/110: 503-508.

MAENO, M., TODATE, A. and KATAGIRI, C. (1985b). The localization of precursor cells for larval and adult hemopoietic cells of Xenopus laevis in two regions of embryos. Dev Growth Diff 27: 137-148.

MILLS, K. R., KRUEP, D. and SAHA, M. S. (1999). Elucidating the origins of the vascular system: a fate map of the vascular endothelial and red blood cell lineages in Xenopus laevis. Dev Bio/209: 352-368.

MOODY, S. A. (1987). Fates of the blastomeres of the 32-cell-stage Xenopus embryo. Dev Biol122: 300-319.

MURAYAMA, E., KISSA, K., ZAPATA, A., MORDELET, E., BRIOLAT, V., LIN, H. F., HANDIN, R. I. and HERBOMEL, P. (2006). Tracing hematopoietic precursor migration to successive hematopoietic organs during zebrafish development. Immunity 25; 963-975.

PALIS, J. (2008). Ontogeny of erythropoiesis. Curr Opin Hemato/15: 155-161.

PATTERSON, L. J., GERING, M., ECKFELDT, C. E., GREEN, A. R., VERFAILLIE, C. M., EKKER, S. C. and PATIENT, R. (2007). The transcription factors Scl and Lmo2 act together during development of the hemangioblast in zebrafish. Blood 109: $2389-2398$.

PATTERSON, L. J., GERING, M. and PATIENT, R. (2005). Scl is required for dorsal aorta as well as blood formation in zebrafish embryos. Blood 105: 3502-3511.

PIMANDA, J. E., OTTERSBACH, K., KNEZEVIC, K., KINSTON, S., CHAN, W. Y., WILSON, N. K., LANDRY, J. R., WOOD, A. D., KOLB-KOKOCINSKI, A., GREEN, A. R. et al. (2007). Gata2, Fli1, and Scl form a recursively wired generegulatory circuit during early hematopoietic development. Proc Nat/ $\mathrm{Acad} \mathrm{SCl}$ USA 104: 17692-17697.

ROLLINS-SMITH, L. A. and BLAIR, P. (1990). Contribution of ventral blood island mesoderm to hematopoiesis in postmetamorphic and metamorphosis-inhibited Xenopus laevis. Dev Biol142: 178-183.

SABIN, F. R. (1920). Studies on the origins of blood-vessels and of red bloodcorpuscles as seen in the living blastoderm of chicks during the second day of development. Contrib Embryo/36: 213-262.

SCHOENEBECK, J. J., KEEGAN, B. R. and YELON, D. (2007). Vessel and blood specification override cardiac potential in anterior mesoderm. Dev Cel/13: 254 267.

SEO, S., FUJITA, H., NAKANO, A., KANG, M., DUARTE, A. and KUME, T. (2006) The forkhead transcription factors, Foxc1 and Foxc2, are required for arterial specification and lymphatic sprouting during vascular development. Dev Biol 294: 458-470.

SHIBATA, T., TAKAHASHI, Y., TASAKI, J., SAITO, Y., IZUTSU, Y. and MAENO, M. (2008). A role of $D$ domain-related proteins in differentiation and migration of embryonic cells in Xenopus laevis. Mech Dev 125: 284-298.

SHIVDASANI, R., MAYER, E. and ORKIN, S. H. (1995). Absence of blood formation in mice lacking the T-cell leukemia oncoprotein tal-1/SCL. Nature 373: 432-434.

SMITH, P. B. and TURPEN, J. B. (1985). Hemopoietic differentiation potential of cultured lateral plate mesoderm explanted from Rana pipiens embryos at successive developmental stages. Differentiation 28: 244-249.

STAINIER, D. Y., WEINSTEIN, B. M., DETRICH, H. W., 3RD, ZON, L. I. and FISHMAN, M. C. (1995). Cloche, an early acting zebrafish gene, is required by both the endothelial and hematopoietic lineages. Development 121: 3141 3150 .

SUMANAS, S., GOMEZ, G., ZHAO, Y., PARK, C., CHOI, K. and LIN, S. (2008). Interplay among Etsrp/ER71, scl and alk8 signaling controls endothelial and myeloid cell formation. Blood 111: 4500-4510.

SUMANAS, S. and LIN, S. (2006). Ets1-related protein is a key regulator of vasculogenesis in zebrafish. PLOS Bio/4: e10.

TAOUDI, S., GONNEAU, C., MOORE, K., SHERIDAN, J. M., BLACKBURN, C. C. TAYLOR, E. and MEDVINSKY, A. (2008). Extensive hematopoietic stem cell generation in the AGM region via maturation of $\mathrm{VE}$-cadherin+CD45+ predefinitive HSCs. Cel/ Stem Cel/3: 99-108.

TASHIRO, S., SEDOHARA, A., ASASHIMA, M., IZUTSU, Y. and MAENO, M. (2006). Characterization of myeloid cells derived from the anterior ventra mesoderm in the Xenopus laevis embryo. Dev Growth Diff 48: 499-512.

TRACEY, W. D., JR., PEPLING, M. E., HORB, M. E., THOMSEN, G. H. and GERGEN, J. P. (1998). A Xenopus homologue of aml-1 reveals unexpected patterning mechanisms leading to the formation of embryonic blood. Develop ment 125: 1371-1380.

TSAI, F. Y., KELLER, G., KUO, F. C., WEISS, M., CHEN, J., ROSENBLATT, M., ALT, F. W. and ORKIN, S. H. (1994). An early haematopoietic defect in mice lacking the transcription factor GATA-2. Nature 371: 221-226.

TURPEN, J. B., KELLEY, C. M., MEAD, P. E. and ZON, L. I. (1997). Bipotential primitive-definitive hematopoietic progenitors in the vertebrate embryo. Immunity 7: 325-334

TURPEN, J. B. and KNUDSON, C. M. (1982). Ontogeny of hematopoietic cells in Rana pipiens: precursor cell migration during embryogenesis. Dev Bio/89: 138151.

TURPEN, J. B., KNUDSON, C. M. and HOEFEN, P. S. (1981). The early ontogeny of hematopoietic cells studied by grafting cytogenetically labeled tissue anlagen: localization of a prospective stem cell compartment. Dev Bio/85: 99-112.

UENO, H. and WEISSMAN, I. L. (2006). Clonal analysis of mouse development reveals a polyclonal origin for yolk sac blood islands. Dev Cel/11: 519-533.

VOGELI, K. M., JIN, S. W., MARTIN, G. R. and STAINIER, D. Y. (2006). A common progenitor for haematopoietic and endothelial lineages in the zebrafish gastrula. Nature 443: 337-339.

WALMSLEY, M., CIAU-UITZ, A. and PATIENT, R. (2002). Adult and embryonic blood and endothelium derive from distinct precursor populations which are differentially programmed by BMP in Xenopus. Development 129: 5683-5695.

WALMSLEY, M., CIAU-UITZ, A. and PATIENT, R. (2005). Tracking and programming early hematopoietic cells in Xenopus embryos. Methods Mol Med 105: 123-136.

WALMSLEY, M., CLEAVER, D. and PATIENT, R. (2008). Fibroblast growth factor controls the timing of $\mathrm{Scl}, \mathrm{Lmo}$, and Runx1 expression during embryonic blood development. Blood 111: 1157-1166.

WANG, C., FALOON, P. W., TAN, Z., LV, Y., ZHANG, P., GE, Y., DENG, H. and XIONG, J. W. (2007). Mouse lysocardiolipin acyltransferase controls the development of hematopoietic and endothelial lineages during in vitro embryonic 
stem-cell differentiation. Blood110: 3601-3609.

WANG, H. U., CHEN, Z. F. and ANDERSON, D. J. (1998). Molecular distinction and angiogenic interaction between embryonic arteries and veins revealed by ephrin-B2 and its receptor Eph-B4. Cel/93; 741-753.

WARREN, A. J., COLLEDGE, W. H., CARLTON, M. B. L., EVANS, M. J., SMITH, A. J. H. and RABBITTS, T. H. (1994). The oncogenic cysteine-rich lim domain protein rbtn2 is essential for erythroid development. Cel/78: 45-57.

WILKINSON, R. N., POUGET, C., GERING, M., RUSSELL, A. J., DAVIES, S. G., KIMELMAN, D. and PATIENT, R. (2009). Hedgehog and Bmp polarize hematopoietic stem cell emergence in the zebrafish dorsal aorta. Dev Cel/16:909-916.
Willett, C. E., CORTES, A., ZUASTI, A. and ZAPATA, A. G. (1999). Early hematopoiesis and developing lymphoid organs in the zebrafish. Dev Dyn214: 323-336.

ZHANG, X. Y. and RODAWAY, A. R. (2007). SCL-GFP transgenic zebrafish: in vivo imaging of blood and endothelial development and identification of the initial site of definitive hematopoiesis. Dev Bio/307: 179-194.

ZOVEIN, A. C., HOFMANN, J. J., LYNCH, M., FRENCH, W. J., TURLO, K. A., YANG, Y., BECKER, M. S., ZANETTA, L., DEJANA, E., GASSON, J. C. et al. (2008). Fate tracing reveals the endothelial origin of hematopoietic stem cells. Cell Stem Cel/3: 625-636.

\section{Further Related Reading, published previously in the Int. J. Dev. Biol.}

See our recent Special Issue Placenta edited by Joan S. Hunt and Kent L. Thornburg at: http://www.ijdb.ehu.es/web/contents.php?vol=54\&issue=2-3

Tracing the hemangioblast during embryogenesis: developmental relationships between endothelial and hematopoietic cells Thierry Jaffredo, Karine Bollerot, Daisuke Sugiyama, Rodolphe Gautier and Cécile Drevon Int. J. Dev. Biol. (2005) 49: 269-277

Of birds and mice: hematopoietic stem cell development Isabelle Godin and Ana Cumano Int. J. Dev. Biol. (2005) 49: 251-257

\section{Embryonic development of the human hematopoietic system}

Manuela Tavian and Bruno Péault

Int. J. Dev. Biol. (2005) 49: 243-250

Pathways in blood and vessel development revealed through zebrafish genetics Philip S Crosier, Maggie L Kalev-Zylinska, Christopher J Hall, Maria Vega C Flores, Julia A Horsfield and Kathryn E Crosier Int. J. Dev. Biol. (2002) 46: 493-502

Two-step induction of primitive erythrocytes in Xenopus laevis embryos: signals from the vegetal endoderm and the overlying ectoderm

M Kikkawa, M Yamazaki, Y Izutsu and M Maéno

Int. J. Dev. Biol. (2001) 45: 387-396

Overexpression of the Xenopus XI-fli gene during early embryogenesis leads to anomalies in head and heart development and erythroid differentiation

P Remy, F Sénan, D Meyer, A M Mager and C Hindelang

Int. J. Dev. Biol. (1996) 40: 577-589


5 yr ISI Impact Factor $(2009)=3.253$



